Dabove, I y Arrubia, E, ¿Demasiado vieja para el sexo? Derecho y Ciencias Sociales. Octubre 2018. № 19. (Las familias y el derecho de las familias a dos años de vigencia del Código Civil y Comercial) Pgs 63-74. ISNN 1852-2971. Instituto de Cultura Jurídica y Maestría en Sociología Jurídica. FCJ y S. UNLP

\title{
¿Demasiado vieja para el sexo?
}

\author{
Too old for sex?
}

\section{Isolina Dabove y Eduardo Arrubia}

\section{Resumen:}

El presente trabajo se propone un análisis del fallo del Tribunal Europeo de Derechos Humanos "Carvalho Pinto de Sousa Morais v. Portugal" a la luz del derecho de la ancianidad y con perspectiva de género. A tal fin primero se hará una introducción sobre algunos aspectos atinentes a la vejez, el género y la sexualidad para posteriormente trabajar sobre los estereotipos presentes en este caso, los aportes que brindan los estudios de género sobre el tema, para finalmente pensar la idea de una justicia restaurativa.

Palabras Claves:Vejez; Sexualidad; Género; Estereotipos.

\begin{abstract}
:
This work intends to analyze the judgment from the European Court of Human Rights named "Carvalho Pinto de Sousa Morais v. Portugal" in the light of regulations aimed at old-aged people with gender perspective. For this purpose, we Will first make an introduction about certain aspects regarding old age,gender and sexuality so as to be able to then work on stereotypes and on the importance of gender perspective research on this subject. Finally,we will address the idea of restorative Justice
\end{abstract}

Key words: Old age; sexuality; gender; sterotype

Isolina Dabove Investigadora del Consejo de Investigaciones Científicas y Técnicas-Universidad de Buenos Aires. Abogada por la Universidad Nacional de Rosario. Doctora en Derecho por la Universidad Carlos III de Madrid. Directora del Centro de investigaciones en Derecho de la Vejez de la Universidad Nacional de Rosario. Directora del Observatorio de Derechos Humanos de las Personas Mayores de la Universidad de Morón. Directora de la Unidad de Investigación en Derecho de la Vejez de la Facultad de Derecho y Ciencias Sociales de la Universidad Nacional de Córdoba. Integrante de la Delegación Oficial Argentina ante la ONU y la OEA en el proceso de elaboración de la Convención Internacional y de la Convención Americana, de Derechos Humanos de las Personas Mayores. CABA, Argentina. isolinadabove@gmail.com

Eduardo Arrubia. Abogado. Becario de Investigación doctoral del Consejo Nacional de Investigaciones Científicas y Técnicas (CONICET). Profesor Adjunto ordinario en la cátedra de Filosofía del Derecho y Ética de la Abogacía (Facultad de Derecho - UNICEN). Doctorando en Derecho (UBA). Miembro del Centro de Estudios en Derechos Humanos (CEDH-UNICEN). E-mail: earrubia@gmail.com

Recibido:11 /6/ 2018 - Aceptado con correcciones: 6/8/2018 
Dabove, I y Arrubia, E, ¿Demasiado vieja para el sexo? Derecho y Ciencias Sociales. Octubre 2018. № 19. (Las familias y el derecho de las familias a dos años de vigencia del Código Civil y Comercial) Pgs 63-74. ISNN 1852-2971. Instituto de Cultura Jurídica y Maestría en Sociología Jurídica. FCJ y S. UNLP

\section{¿Demasiado vieja para el sexo?}

\section{Isolina Dabove y Eduardo Arrubias}

Goza sin temor del hado, el curso breve de tu edad lozana, pues no podrá la muerte de mañana quitarte lo que hubieres hoy gozado; y aunque llega la muerte presurosa $Y$ tu fragante vida se te aleja, no sientas el morir tan bella y moza: Mira que la experiencia te aconseja Que es fortuna morirte siendo hermosa $Y$ no ver el ultraje de ser vieja.

SOR JUANA INES DE LA CRUZ

Soneto 148

\section{Vejez, género y sexualidad}

La condición jurídica de la mujer ha sido objeto de análisis y reflexión de manera muy reciente. En efecto, solo a partir del siglo XIX fue posible el desarrollo de los primeros movimientos políticos para el reconocimiento de sus derechos. Así como también solo desde entonces comenzaron a plantearse teorías feministas y estudios de género y diversidad sexual. Sin embargo, este corto recorrido ha sido tan fructífero y contundente que ahora nos permite abordar cuestiones aún vedadas al imaginario colectivo.

Una de las nuevas problemáticas abiertas por este proceso es, sin dudas, la comprensión jurídica de la mujer, en cuyo marco, los derechos sexuales y reproductivos han adquirido hoy, un claro protagonismo. Pero de otro también lo es la discusión en torno al estatus jurídico de la mujer anciana, a las particularidades que atraviesan su vida y sus derechos y a las condiciones que le imposibilitan su ejercicio. 
Dabove, I y Arrubia, E, ¿Demasiado vieja para el sexo? Derecho y Ciencias Sociales. Octubre 2018. № 19. (Las familias y el derecho de las familias a dos años de vigencia del Código Civil y Comercial) Pgs 63-74. ISNN 1852-2971. Instituto de Cultura Jurídica y Maestría en Sociología Jurídica. FCJ y S. UNLP

Muchos y pesados son los prejuicios y estereotipos que suelen ser atribuidos a las mujeres mayores de hoy en día, tanto como larga y densa es su tradición. Tal como hemos observado en anteriores investigaciones, la desvalorización de las mujeres de edad avanzada estaba presente tanto en la condición de "alieni iuris" que les otorgaba el derecho romano antiguo, como en los bajísimos montos indemnizatorios vigentes en el derecho germano medieval establecidos sobre ellas a causa de la pérdida del poder reproductivo.

Hay un hecho muy curioso entre los bárbaros, que ilustra esta devaluación de las mujeres viejas: la compensación pecuniaria exigida por el asesinato de un hombre libre, el wergeld. En el siglo VI, el Derecho visigótico reclamaba: "60 centavos oro por un niño de un año; 150 centavos oro por un muchacho de 15 a 20 años; 300 centavos oro por un hombre de 20 a 25 años; 200 centavos oro por un hombre de 50 a 65 años; 100 centavos oro por un hombre de más de 65 años; 250 centavos oro por una mujer de 15 a 40 años; 200 centavos oro por una mujer de 40 a 60 años"1. En el Derecho burgundio la suma era de: "300 centavos oro, entre 20 y 50 años; 200 centavos oro, entre 50 y 65 años; 150 centavos oro, después de los 65 años" (Beauvoir 1989,152).

Entre los francos, por el homicidio de una mujer embarazada o madre de familia, había que pagar una compensación tres veces mayor que la del precio del varón; hasta la menopausia y muy poco después. En efecto, quien matase a una mujer joven y libre, en edad de procrear, tenía que pagar 600 sueldos. Mientras que, si la mujer moría después de la menopausia, sólo tenía que abonar 200. Si se le hería estando embarazada y moría, correspondían 700 sueldos de multa; pero se reducía a 100 si el niño moría a consecuencia del aborto consiguiente ${ }^{2}$. Esta desvalorización jurídica también quedó de manifiesto en la asociación acrítica de las mujeres mayores con su condición de brujas, esperpentos, viejas locas, o alcahuetas, eficazmente instalada por filósofos, artistas, literatos y juristas (Dabove 2002 y 1996).

Entre las imágenes más descarnadas se encuentran las escritas por Erasmo de Roterdam quien logra sintetizar los prejuicios renacentistas ${ }^{3}$. En Elogio de la locura, por ejemplo, señala que:

\footnotetext{
${ }^{1}$ Entre las mujeres, la función reproductora es la que marca la diferenciación en la cotización. Al respecto ver: BEAUVOIR (1989) Y CASEY (1989).

${ }^{2}$ RouCHE, (1992) Cuenta que "hacia fines del siglo VI, el rey Gontran promulgó una estipulación suplementaria, probablemente porque este género de delito se estaba multiplicando: en adelante había que pagar 600 sueldos por una mujer encinta asesinada, y, encima, otros 600 si el niño muerto iba a ser un varón”.

${ }^{3}$ En este sentido, y muy agudamente señala BEAUVOIR (1989:179 Y 1989:183) "El prejuicio antifeminista contra las mujeres de edad es evidente en Erasmo. Es normal que este moralista repruebe a las que tienen la indecencia de pensar todavía en el amor. Pero la maldad gratuita de su descripción sorprende en un humanista. Innumerables son también los poetas renacentistas que cubren de oprobio a la mujer de edad; verbigracia, Marot, Desportes, Du Bellay, Sigonio o Hulot. Tan sólo un autor asumió vivamente la defensa de las mujeres de edad: Brantôme en la
} 
Dabove, I y Arrubia, E, ¿Demasiado vieja para el sexo? Derecho y Ciencias Sociales. Octubre 2018. № 19. (Las familias y el derecho de las familias a dos años de vigencia del Código Civil y Comercial) Pgs 63-74. ISNN 1852-2971. Instituto de Cultura Jurídica y Maestría en Sociología Jurídica. FCJ y S. UNLP

mucho más gracioso todavía, es ver a ciertas ancianas que apenas pueden con el peso de sus años y semejan cadáveres que se diría han vuelto del infierno. Van diciendo siempre "qué bella es la luz"; siguen estando calientes y, según dicen los griegos, "como cabras en celo" buscan a gran precio conquistar algún joven Faón. A este fin, maquillan exageradamente su cara, no se apartan nunca del espejo, depilan el monte de venus, hacen ostentaciones de sus pechos lacios y ajados, con voz trémula e insinuante tratan de hacer revivir una pasión que se extingue, beben, bailan entre las jovencitas, y hasta escriben pequeñas cartas de amor. (1989:70)

Y más adelante agrega: "Todos se ríen de estas cosas, como tonterías supinas que son. Pero, mientras tanto, estas ancianas viven satisfechas y contentas, nadan en delicias, la vida es pura miel, y me deben a mí su felicidad (Erasmo de Rotterdam, 1989:70).

Por fortuna desde fines del siglo XIX, la cultura ha comenzado a poner en entredicho estas significaciones en todos sus campos, permitiendo el desarrollo de instrumentos humanistas de empoderamiento y transformación social. Un logro jurídico representativo de estos nuevos recorridos es el caso que seguidamente analizaremos, protagonizado por una mujer mayor que fue objeto de discriminación múltiple respecto de sus derechos sexuales a causa de su edad y género ( Thomsons Trade Union Solicitors, 2017).

\section{Un caso paradigmático}

La Sra. Maria Ivone Carvalho Pinto de Sousa Morais, nacida en 1945 en Portugal, padecía una enfermedad ginecológica a causa de la cual tuvo que ser intervenida quirúrgicamente en el año 1995. Como resultado de esta operación, a los 50 años de edad la demandante quedó en una situación de intenso dolor, sumado a la pérdida de sensibilidad vaginal, incontinencia, dificultad de locomoción, e imposibilidad de mantenimiento de relaciones sexuales. Posteriormente, se comprobó que durante la intervención se había afectado el nervio pudendo lo cual constituía un hecho evidente de mala praxis médica. Esto motivó que la Sra. de Sousa Morais incoara una acción de daños contra el hospital y que llegara el 27 de julio de 2017 al Tribunal Europeo de Derechos Humanos, en caso titulado "Carvalho Pinto de Sousa Morais v. Portugal".

En octubre de 2013, la primera instancia judicial falló a su favor y ordenó al hospital a pagarle una indemnización de ochenta mil euros en concepto de daños físicos y mentales originados en el error médico y dieciséis mil euros para afrontar los gastos de una persona que le preste

\footnotetext{
"Vie des dames galantes". Es el único que considera normal que las mujeres se entreguen todavía a los placeres del amor. Incluso es el único que llega a reconocer que algunas siguen siendo bellas y amadas después de los setenta años". También ver ALBA (1992:68)
} 
ayuda en el hogar, debido a que la mujer había quedado impedida de realizar tareas domésticas por sí misma. Sin embargo, un año después, la Suprema Corte Administrativa de Portugal redujo esta compensación a un tercio. Entre otras razones, entendió que a pesar de estar probados los hechos constitutivos de mala praxis médica, las indemnizaciones fijadas por el a quo eran desproporcionadas y excesivas. El Tribunal argumentó que, si bien el sufrimiento de la actora había sido agravado por la intervención quirúrgica, este cuadro era preexistente a dicha operación. Por otro lado, subrayó que al momento de la cirugía la mujer ya tenía 50 años de edad, era madre de dos hijos adultos y sólo debía prestar tareas del hogar a favor de su marido, todo lo cual restaba importancia al ejercicio de su sexualidad.

Frente a esta decisión, la actora llevó su caso al Tribunal Europeo de Derechos Humanos (TEDH), alegando que había sido discriminada debido a su género y de su mayor edad. Luego de un año y medio de tramitación, Estrasburgo se expidió en su favor, pero por mayoría de votos. Señalaron que los jueces portugueses se habían apoyado en prejuicios estereotipados acerca del rol de la mujer en la sociedad. Pero, además, reconocieron que lo reprochable de las anteriores soluciones radicaba en el hecho de haber considerado que la sexualidad no es tan importante para una mujer de 50 años y madre de dos que para alguien más joven. En concreto, expresaron que: "este postulado refleja la idea tradicional de la sexualidad femenina como algo esencialmente vinculado a propósitos reproductivos y por lo tanto ignora su importancia física y psicológica para la realización de las mujeres como personas" (Oddny Mjoll, 2017).

\section{Sobre estereotipos y estragos jurídicos}

En el caso bajo análisis puede advertirse como un hecho probado la discriminación negativa que padeció la demandante. Esta situación se comprende mejor si se tiene en cuenta que la edad avanzada se utiliza como un elemento prejuicioso que menoscaba la dignidad de la persona, cuando en rigor debería operar como una coyuntura vital en la que los derechos no se pierden, aunque éstos requieran una tutela especial. En este sentido, es necesario comprender que la vejez es un fenómeno bio-psico-social, es decir, que no existe una edad decisiva para establecer cuándo una persona requiere una protección especial de sus derechos en relación a la vulnerabilidad resultante de su avanzada edad ${ }^{4}$. Por el contrario, la condición de ancianidad de la persona es siempre una construcción social, cultural, histórica y política ( Dabove y Pronotto Laborde, 2006:11). Es por ello que desde la gerontología se ha empezado a hablar de

\footnotetext{
${ }^{4}$ No obstante, corresponde decir que las normas jurídicas establecen una edad límite a partir de la cual el individuo es considerado como adulto mayor. Por ejemplo, la Convención Interamericana sobre la Protección de los Derechos Humanos de las Personas Mayores define a la persona mayor como aquella de 60 años o más, salvo que la ley interna determine una edad base menor o mayor, siempre que ésta no sea superior a los 65 años.
} 
Dabove, I y Arrubia, E, ¿Demasiado vieja para el sexo? Derecho y Ciencias Sociales. Octubre 2018. № 19. (Las familias y el derecho de las familias a dos años de vigencia del Código Civil y Comercial) Pgs 63-74. ISNN 1852-2971. Instituto de Cultura Jurídica y Maestría en Sociología Jurídica. FCJ y S. UNLP

envejecimientos en plural para denotar la complejidad y heterogeneidad que caracteriza al último ciclo vital de una persona (Dabove 2015:12).

Ante este panorama, el derecho de la vejez emerge en función de que la persona anciana se halla en una triple situación de vulnerabilidad (Dabove 2015:14) que claramente puede individualizarse a título ejemplificativo en el caso que aquí se comenta. De esta manera, la demandante fue expuesta a una vulnerabilidad sociológica en el sentido de que los prejuicios y estereotipos imperantes en la sociedad portuguesa determinaron que se entendiera que una mujer de 50 años de edad no necesita de sus funciones biológicas sexuales dado que ya había sido madre y su ciclo reproductivo estaba cumplido.

También aparece una vulnerabilidad normológica ya que no existían normas jurídicas que la ampararan y que constriñeran a los jueces a respetar los derechos humanos de los que son titulares todas las personas en este momento de la vida. Simultáneamente, se da una vulnerabilidad axiológica ya que la persona no es valorada como un fin en sí misma sino que es mediatizada en función de otros. Esto se advierte claramente en el argumento que utilizan los jueces del Superior Tribunal Administrativo de Portugal para reducir la indemnización de cobertura de gastos por tareas domésticas. Esto es, se sostiene la idea de que la mujer, cuyos hijos se encuentran en una edad adulta, debe asistir con servicios domésticos a su marido. La mujer es tomada como un medio para la satisfacción de las necesidades de su marido-hombre.

Ahora bien, aun cuando sobre este punto en particular la disminución del rubro indemnizatorio por gastos de servicio doméstico pueda llegar a justificarse, entendemos que no lo era por vía del argumento utilizado. En efecto, esta reducción podría haberse fundado señalando que el monto pecuniario sólo debería cubrir aquellas tareas domésticas y de cuidado que la demandante estaba imposibilitada de realizar por sí y para ella misma, a causa de la mala praxis médica. De esta manera, no sólo los hijos adultos, sino también su marido podrían haberse hecho cargo de sus necesidades y de las tareas hogareñas vinculadas al mantenimiento de sus vidas cotidianas.

Así, pues, en el caso se registran los prejuicios ancestrales en relación a la sexualidad femenina en la vejez. Desde el propio tribunal portugués de alzada se argumenta que la indemnización por daños físicos y psíquicos debe ser reducida ya que la afectación de su genitalidad a raíz de la mala praxis no es considerada tan significativa debido a la avanzada edad de la mujer. Esto encubre la idea de que la función sexual de la mujer existe en el cuerpo humano a los efectos de la procreación únicamente, y que su destino biológico es el de ser madre. Claramente se constituye aquí una visión estereotipada de la sexualidad femenina per se y, al mismo tiempo, de la sexualidad en la vejez. Esto es así ya que desde una mirada integral la salud sexual de un individuo no sólo comprende el binomio sexo-reproducción sino que además incluye el de sexo-placer (Maffia y Cabral 2003: 88). 
Dabove, I y Arrubia, E, ¿Demasiado vieja para el sexo? Derecho y Ciencias Sociales. Octubre 2018. № 19. (Las familias y el derecho de las familias a dos años de vigencia del Código Civil y Comercial) Pgs 63-74. ISNN 1852-2971. Instituto de Cultura Jurídica y Maestría en Sociología Jurídica. FCJ y S. UNLP

De la misma manera, los prejuicios acechan a la sexualidad en la ancianidad ya que se estigmatiza al adulto mayor por medio del entendimiento de que ya no es apto para mantener relaciones sexuales debido a su avanzada edad. Así, se piensa al viejo a través de una discapacidad fáctica para lo sexual, y este pensamiento es funcional a una lógica que "patologiza" la vejez (Iacub, 2011:132). En contra de semejante visión estereotipada se ha sostenido que "las distintas transformaciones fisiológicas que la vejez traiga aparejada podrán habilitar un discurso que contemple casos médico-clínicos tendientes a resguardar la salud del anciano, pero de ninguna manera podrán constituir un elemento que legitime un discurso jurídico de negación en cuanto a la titularidad y ejercicio de los derechos sexuales de la persona anciana". (Arrubia 2016:78)

\section{Relevancia jurídica de la diversidad}

Tradicionalmente, el desarrollo de las teorías feministas ha significado la afirmación de la existencia del binomio hombre-mujer. Si bien esta contrastación se ha encauzado en términos de emancipación de la segunda respecto del primero con exitosa gradualidad, lo cierto es que este antagonismo presenta ciertas limitaciones a la reflexión sobre la mujer en los tiempos que corren (De Laurentis 1987:1). Es decir, se reconoce en la formulación de dicho par dicotómico un aspecto positivo y otro negativo. El aspecto positivo aparece porque este ideal de mujer autónoma, emancipada del hombre, constituye una herramienta fuerte para lograr cohesión social a través de la identificación común posibilitada por el reconocimiento de enlace entre los individuos de una comunidad. Pero, el sentido negativo también está representado allí, porque ese ideal puede erigirse como una amenaza para esa misma cohesión social cuando se lo hace funcionar como mecanismo de exclusión de una parte de la comunidad (Araujo, 2009: 8). De esta manera, para el feminismo, dicha conceptualización por oposición impide pensar el rol o la situación de la mujer en función la mujer misma.

Entonces, ¿Serán iguales las necesidades y los consiguientes derechos de una mujer rica en comparación con una mujer pobre, los de una mujer joven en relación con una mujer anciana, los de una mujer heterosexual en comparación con una mujer lesbiana? Claramente el escenario de diferencias que se agudizan dentro del mismo grupo vulnerable conformado por mujeres ha exigido que los enfoques teóricos empiecen a girar en torno a estas cuestiones. Por lo tanto, desde este lugar debe procederse a la deconstrucción de la categoría género a los efectos de correr el foco de análisis desde la tradicional búsqueda de igualdad de la mujer respecto del hombre. Pero de-construir implica al mismo tiempo reconstruir. Entonces, siguiendo a De Laurentis, nos preguntamos si la deconstrucción de género inevitablemente produce su reconstrucción, la pregunta que emerge es ¿en qué términos y en interés de quiénes es producida la de-reconstrucción? (1987:6). 
Dabove, I y Arrubia, E, ¿Demasiado vieja para el sexo? Derecho y Ciencias Sociales. Octubre 2018. № 19. (Las familias y el derecho de las familias a dos años de vigencia del Código Civil y Comercial) Pgs 63-74. ISNN 1852-2971. Instituto de Cultura Jurídica y Maestría en Sociología Jurídica. FCJ y S. UNLP

Por otro lado, se ha sostenido también que "la idea de igualdad no debe ser ni ignorada ni suprimida del análisis, sino que debe buscarse la manera de integrar y armonizar este ideal con el abordaje de las profundas diferencias y heterogeneidades que aparecen en el tejido social real" (Arrubia 2016, 19). De modo tal que, a nuestro criterio, no es posible arrogarse la facultad de situarse a favor de la diferencia y en contra de la universalidad de una forma radical y absoluta.

En este punto, resulta interesante advertir que el caso judicial que aquí se analiza representa ambos sentidos del feminismo en comunión. Esto es, por un lado, el TEDH condena estereotipos que están vinculados al patriarcado, a la subyugación del rol de la mujer frente al hombre a través de la consideración de elementos de un sistema social patriarcal tales como el ejercicio de la sexualidad y el trabajo doméstico (Walby 1990: 25). Por otro lado, simultáneamente entiende que la discriminación aparece agravada por estar vinculada a la edad de la mujer, lo cual plantea un terreno de especificidad diferente del de una mujer joven. Es decir, se piensa en la corroboración de diferencias al interior del mismo grupo vulnerable reivindicado desde el feminismo ya que éstas exigen una respuesta judicial ad hoc. De esta manera, según Fraser, el reconocimiento desde el derecho no debe estar orientado a la especificidad propia de un grupo, en este caso el de mujeres, sino al estatus de los miembros individuales de un grupo como plenos participantes de la interacción social (2000: 61).

Paralelamente, en materia de sexualidad se han construido nuevos estándares internacionales desde el soft law a fines del año 2017 que sirven para encuadrar el caso en análisis. Así, con relación a la diversidad sexual, se han fijado ciertos estándares de derechos humanos a través de los Principios de Yogyakarta sobre la Aplicación de la Legislación Internacional de Derechos Humanos en relación con la Orientación Sexual y la Identidad de Género de 2006.

Estos principios se originaron con una función tripartita, esto es, la de relevar las experiencias reales de discriminación del colectivo LGBTI en todas las regiones del mundo, la de articular de manera clara y precisa la aplicación de la legislación internacional de Derechos Humanos a dichas experiencias, y la de establecer la naturaleza de las obligaciones que deben asumir los Estados ante estas violaciones (O’Flaherty y Fisher, 2008: 233). Si bien este documento integra el denominado soft law, es decir aquel derecho internacional que no es vinculante por sí mismo, lo cierto es que constituye una serie de pautas hermenéuticas valiosas a la hora de interpretar la normativa internacional vinculante. En este sentido, corresponde contrastar dos conceptos similares, pero diferentes. Por un lado, la noción de diversidad sexual se refiere a las diversas manifestaciones de la sexualidad humana, dentro de la cual tiene lugar la heterosexualidad. Por el otro, la idea de disidencia sexual (Salinas Hernández 2006: 23) alude a esta variedad de sexualidades, pero excluyendo a la heterosexualidad ya que se considera que ésta ha sido una expresión hegemónica en términos históricos y culturales. 
Dabove, I y Arrubia, E, ¿Demasiado vieja para el sexo? Derecho y Ciencias Sociales. Octubre 2018. № 19. (Las familias y el derecho de las familias a dos años de vigencia del Código Civil y Comercial) Pgs 63-74. ISNN 1852-2971. Instituto de Cultura Jurídica y Maestría en Sociología Jurídica. FCJ y S. UNLP

La disidencia sexual se traduce como una reivindicación con sentido político de aquellas sexualidades oprimidas frente a la hegemonía de la heteronormatividad. En este orden de ideas, los principios mencionados pueden ser concebidos como un marco de mandatos vinculados a la diversidad sexual debido a que a pesar de tener primacía los derechos de la homosexualidad y la "transgeneridad" también trascienden en importancia respecto de situaciones discriminatorias relacionadas con la heterosexualidad. Esto queda reafirmado con la actualización que se dio a estos principios a fines de 2017.

En esta ocasión, la reunión de expertos auspiciada por la Organización de Naciones Unidas receptó prima facie las mismas categorías sospechosas de discriminación negativa del instrumento originario (orientación sexual, identidad de género y expresión de género) pero además incorporó una nueva, esto es, las características sexuales. Las mismas se han definido como las "características físicas relacionadas con el sexo, incluyendo la genitalidad como así también cualquier otra parte sexual y reproductiva de la anatomía, cromosomas, hormonas y caracteres físicos secundarios que surgen en la pubertad".5. Por lo tanto, resulta de crucial importancia incorporar a este hilo argumentativo el principio 32 resultante del mencionado proceso de actualización de este instrumento, referido al derecho a la integridad corporal y mental.

Así, su texto dispone que: "Todas las personas tienen el derecho a la integridad corporal y mental, a la autonomía y a la autodeterminación independientemente de la orientación sexual, la identidad de género, la expresión de género y las características sexuales. Nadie estará sujeto a procedimientos médicos invasivos o irreversibles que modifiquen sus características sexuales sin su consentimiento previo, libre e informado, a menos que sea necesario evitar un daño serio, urgente e irreparable a la persona" ${ }^{\text {. }}$.

Por consiguiente, esta afortunada inclusión de las características sexuales al elenco de categorías sospechosas de discriminación permite proteger los cuerpos de personas intersexuales o hermafroditas, repeliendo todo tipo de intervención médica injustificada desde el nacimiento. Pero también en el ámbito de la heterosexualidad permite rechazar hechos discriminatorios hacia una mujer sobre cuya genitalidad se juzgue una ineptitud para el mantenimiento de relaciones sexuales con su marido en función de la edad.

\section{Hacia una justicia restaurativa}

El Tribunal Europeo de Derechos Humanos acogió el reclamo de la demandante por vía del reconocimiento de la existencia de los prejuicios y estereotipos aquí reseñados. De esta

\footnotetext{
${ }^{5}$ VV.AA, The Yogyakarta Principles plus ten, (2017: 6)

${ }^{6}$ VV.AA, The Yogyakarta Principles plus ten, (2017:10).
} 
Dabove, I y Arrubia, E, ¿Demasiado vieja para el sexo? Derecho y Ciencias Sociales. Octubre 2018. № 19. (Las familias y el derecho de las familias a dos años de vigencia del Código Civil y Comercial) Pgs 63-74. ISNN 1852-2971. Instituto de Cultura Jurídica y Maestría en Sociología Jurídica. FCJ y S. UNLP

manera, el TEDH sentenció que el Estado de Portugal había violado el art. 8 del Convenio Europeo de Derechos Humanos en el sentido de que la sentencia del tribunal de alzada constituyó una clara intervención ilegítima en la vida privada y familiar de la demandante al intentar determinar las características de su plan de vida sexual.

Además, es importante destacar que el TEDH entendió que esta trasgresión se dio en conjunto con la violación del art. 14 del mencionado Convenio ya que se trata de un caso de discriminación negativa múltiple al estar implicadas categorías tales como el sexo femenino de la mujer junto con su avanzada edad. De esta manera, la sentencia resulta ejemplar a los efectos de de-construir estereotipos arraigados en las sociedades que causan daño a la persona en tanto limitan la autonomía y la dignidad del ser humano.

Nuestra región, por su parte, también ha sabido avanzar en esta dirección, tal como lo acredita la Convención Interamericana sobre la protección de los derechos humanos de las personas mayores. En este sentido, un lugar destacado lo ocupa el deber de trabajar en favor de la igualdad y no discriminación y el especial reconocimiento del derecho a la equidad e igualdad de género y enfoque de curso de vida, entre sus principios rectores ${ }^{7}$.

Estamos, pues, a las puertas de un nuevo paradigma. Ha llegado el momento de asumir la diversidad y la vejez a partir de una cultura jurídica basada en el principio de la esperanza. Esperanza que nos sitúa siempre sobre el miedo, que no es pasiva, como éste, ni, menos aún, está encerrada en un anonadamiento, en palabras de Bloch. Ha llegado, en suma, el momento de salir del encierro de los mitos y prejuicios y de entregarnos activamente al proceso de este devenir al que cada uno de nosotros, pertenece, como logró hacerlo el propio Tribunal Europeo de Derechos Humanos frente a los derechos de una mujer, como el de tantas otras, Maria Ivone Carvalho Pinto de Sousa Morais (Bloch, 2004:25).

\section{Referencias Bibliográficas}

Alba, V. (1992). Historia social de la vejez. Barcelona. Laertes. P. 68.

Araujo, K. (2009). Individuo y feminismo. Notas desde América Latina. Íconos. Revista de Ciencias Sociales, Nro. 33. P. 8

\footnotetext{
${ }^{7}$ Organización de Estados Americanos, Convención Interamericana sobre la protección de los derechos humanos de las personas mayores, artículo 3 . Disponible http://www.oas.org/es/sla/ddi/tratados_multilaterales_interamericanos_a70_derechos_humanos_personas_mayores.asp
} 
Dabove, I y Arrubia, E, ¿Demasiado vieja para el sexo? Derecho y Ciencias Sociales. Octubre 2018. № 19. (Las familias y el derecho de las familias a dos años de vigencia del Código Civil y Comercial) Pgs 63-74. ISNN 1852-2971. Instituto de Cultura Jurídica y Maestría en Sociología Jurídica. FCJ y S. UNLP

Aries, P. (1998). Las pretensiones universales del pensamiento político, en Barret, M. y Aries, P. (comp.), Desestabilizar la teoría. Debates Feministas Contemporáneos, México, UNAM - PAIDOS. P. 12.

Arrubia, E. (2016A). Viejos, Humanos y Sexuales. Una reivindicación jurídico - antropológica de la sexualidad en la vejez, Revista Intersercciones en Comunicación, Nro. 9, UNCPBA, Olavarría. P. 78.

Arrubia, E. (2016B). Iguales o diferentes? Los derechos de las personas LGBTI en discusión. Revista de la Facultad de Derecho, Nro. 41, Universidad de la República, Uruguay. P. 19. Disponible en http://revista.fder.edu.uy/index.php/rfd/article/view/554/814 $(6 / 6 / 2018)$

Beauvoir, S. (1989). La vejez. Barcelona. Edhasa. P. 151

Bloch, E. (2004). El Principio Esperanza, Serra, F. (ed) Madrid. Trotta. Vol. I. P. 25.

Casey, J. (1989). Historia de la Familia. Madrid. Espasa-Calpe. P. 53.

Dabove, M. I. (1996). Los derechos de los ancianos. Buenos Aires. Ciudad Argentina.

Dabove, M.I. (2002). La condición jurídica de la mujer anciana, en Bioética y Bioderecho. P. 49 a 54.

Dabove, M. I. (2015). De los derechos humanos al derecho de la vejez en Dabove, M. I. (dir), Derechos humanos de las personas mayores. Acceso a la justicia y protección internacional, Astrea, Buenos Aires, 2015, p 12.

Dabove, M. I. y Prunotto, Laborde, A. (2006). Derecho de la Ancianidad. Perspectiva Interdisciplinaria. Rosario. Editorial Juris. P. 11

De Laurentis, T. (1987). Technologies of gender. Essays on theory, film and fiction, Indiana. Indiana University Press. P. 1 y 6.

De Rotterdam, E. (1989). Elogio de la locura. Madrid. Alianza, Colección El libro de Bolsillo. P. 70 .

Fraser, N. (2000). Nuevas reflexiones sobre el reconocimiento, "New Left Review", Madrid. P. 61.

Iacub, R. (2011). Erótica y vejez. Perspectiva de occidente. Buenos Aires. Paidós. P. 132

Maffia, D. y Cabral, M. (2003). Los sexos, ¿son o se hacen?, en Maffia, D. (comp.) Sexualidades migrantes. Género y tansgénero. Buenos Aires. Feminaría Editora. P. 88. 
Dabove, I y Arrubia, E, ¿Demasiado vieja para el sexo? Derecho y Ciencias Sociales. Octubre 2018. № 19. (Las familias y el derecho de las familias a dos años de vigencia del Código Civil y Comercial) Pgs 63-74. ISNN 1852-2971. Instituto de Cultura Jurídica y Maestría en Sociología Jurídica. FCJ y S. UNLP

Oddny Mjoll, A. (2017). Vulnerability under Article 14 of the European Convention on Human Rights Innovation or Business as Usual?En Juriidca, Årgang 4, Nr. 3, 14 de diciembre de 2017. P- 150 a 171. Disponible en: https://juridika.no/faglitteratur/tidsskrifter/oslo-lawreview/2017/3/artikkel/arnard\%C3\%B3ttir (6/6/2018)

O’ Flaherty, M y Fisher, J (2008). Sexual orientation, Gender identity, and International Human Rights Law: Contextualizing the Yogyakarta Principles. Human Rights Law Review, Vol. 8:2, Oxford University Press. P. 233.

Organización de Estados Americanos.Convención Interamericana sobre la protección de los derechos humanos de las personas mayores, artículo 3. Disponible en: http://www.oas.org/es/sla/ddi/tratados_multilaterales_interamericanos_a70_derechos_humanos_personas_mayores.asp (6/6/2018).

Rouche, M. (1992). Alta Edad Media Occidental: La violencia y la muerte. Sagrado y secretos. En Aries, P. y Duby, G.. Historia de la Vida Privada. Madrid. Taurus. P. 53.

Salinas Hernández, H. M. (2006). Políticas públicas de disidencia sexual: apuntes para una agenda. En Consejo Nacional para prevenir la discriminación, Disidencia sexual e identidades sexuales y genéricas. México. Consejo Nacional para Prevenir la Discriminación (Conapred). P. 23.

Thompsons Trade Union Solicitors, Too old for sex, en Labour \& European Law Review, Weekly issue 535, 30 August 2017. Disponible en; https://www.thompsonstradeunion.law/news/lelr/weekly-issue-535/too-old-for-sex $(6 / 6 / 2018)$

VV.AA, The Yogyakarta Principles plus ten, 2017, p. 6. Disponible en http://yogyakartaprinciples.org/wp-content/uploads/2017/11/A5_yogyakartaWEB-2.pdf

VV.AA (2017), The Yogyakarta Principles plus ten. P. 10

Walby S. (1990), Theorizing patriarchy, New York. Basil Blackwell. P. 25.

\section{Jurisprudencia}

Caso "Carvalho Pinto de Sousa Morais v. Portugal", del 27 de julio de 2017. Disponible en: http://www.humanrightseurope.org/2017/08/portugal-botched-surgery-victim-winscourt-backing-in-gender-and-ageism-human-rights-complaint/(2/4/2018) 